\title{
Systemic View on Host-Pathogen Interactions
}

\author{
R. Reimer, O. Bruns, B. Holstermann, M. Warmer and H. Hohenberg.
}

Electron Microscopy and Micro-Technology, Heinrich-Pette-Institute for experimental Virology and Immunology at the University of Hamburg, Germany

The use of infected 3D culture cells or tissue systems, environmental- and cryopreparation techniques and 3D electron microscopy (EM) combines techniques and methods ideal for the systemic analysis of lifelike host-pathogen interaction on the ultrastructural level.

The best available approach so far to preserve the vital state of infected cells in situ is a chain of cryopreparation steps starting with cryofixation, followed by freeze-substitution, low-temperature embedding and polymerisation. In the case of infected tissues or 3D cell culture networks, only highpressure freezing permits adequate cryoimmobilisation in milliseconds up to a thickness of $200 \mu \mathrm{m}$ that corresponds to approx. 40 cell layers of a 3D culture. Ultrathin sections of such samples, investigated by tomographic electron microscopy allow the structural analysis and 3D reconstruction of the interaction between host cells and pathogens (viruses, bacteria and parasites) in a "lifelike" state inside of a complex cytoplasmic environment that was immobilized in milliseconds and completely cryoprocessed. It is accepted that cryopreparation techniques avoid the typical preparation artefacts induced by conventional EM-preparation techniques based on chemical fixation and total dehydration at room temperature. The conventional preparation steps change for instance the intracellular $\mathrm{pH}$ and the ion equilibrium, which affects the pathogens as well as the structural elements of the host cell involved in the pathogen-cell interaction processes like uptake, intracellular transport, elimination or reproduction and release. Therefore a reliable, reproducible and precise correlation between structural and functional aspects of the interaction between host and pathogen is difficult at least if conventional preparation techniques are used. Cryopreparation by contrast preserves the structural integrity of both the host and the pathogen thereby allowing the analysis of intact and complex lifelike preserved infected biosystems.

This research is supported by the Federal Ministry of Education and Research (BMBF). The aid of Dr. Michael Winkler (Institute for Infection Medicine, Kiel, Germany) is gratefully acknowledged. 


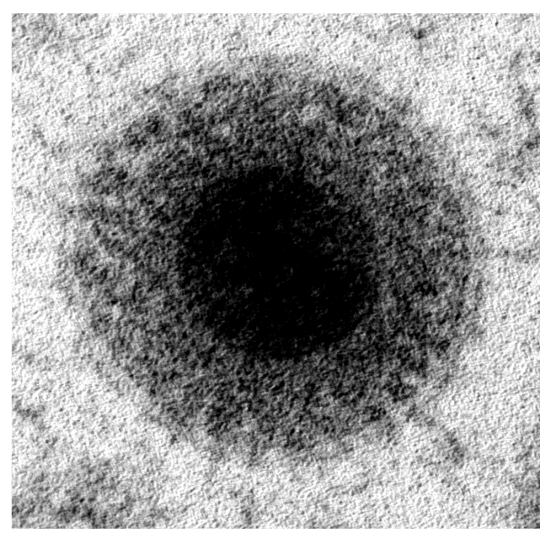

FIG. 1. Preservation of cytomegalovirus structure after environmental- and cryopreparation.

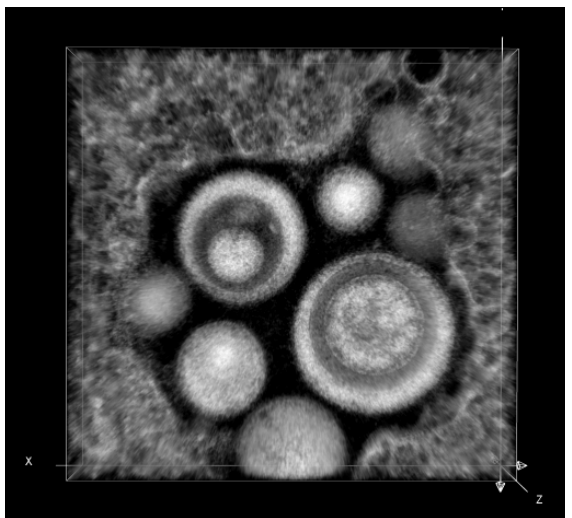

FIG. 2. 3D-reconstruction of the different steps of cytomegalovirus maturation investigated in situ. Box edge length $800 \mathrm{~nm}$. 